\title{
Electrostatic modes in multi-ion and pair-ion collisional plasmas
}

\author{
J. Vranjes, ${ }^{1,2}$ D. Petrovic, ${ }^{3}$ B. P. Pandey, ${ }^{4}$ and S. Poedts ${ }^{1}$ \\ ${ }^{1}$ Center for Plasma Astrophysics, and Leuven Mathematical Modeling and Computational Science Center \\ (LMCC), Celestijnenlaan 200 B, 3001 Leuven, Belgium \\ ${ }^{2}$ Faculté des Sciences Appliquées, Avenue F.D. Roosevelt 50, 1050 Bruxelles, Belgium \\ ${ }^{3}$ Research Group PLASMANT, Department of Chemistry, University of Antwerp, \\ Universiteitsplein 1, 2610 Antwerp, Belgium \\ ${ }^{4}$ Department of Physics, Macquarie University, Sydney, NSW 2109, Australia
}

(Received 11 March 2008; accepted 30 May 2008; published online 8 July 2008)

\begin{abstract}
The physics of plasmas containing positive and negative ions is discussed with special attention to the recently produced pair-ion plasma containing ions of equal mass and opposite charge. The effects of the density gradient in the direction perpendicular to the ambient magnetic field vector are discussed. The possible presence of electrons is discussed in the context of plasma modes propagating at an angle with respect to the magnetic field vector. It is shown that the electron plasma mode may become a backward mode in the presence of a density gradient, and this behavior may be controlled either by the electron number density or the mode number in the perpendicular direction. In plasmas with hot electrons an instability may develop, driven by the combination of electron collisions and the density gradient, and in the regime of a sound ions' response. In the case of a pure pair-ion plasma, for lower frequencies and for parameters close to those used in the recent experiments, the perturbed ions may feel the effects of the magnetic field. In this case the plasma mode also becomes backward, resembling features of an experimentally observed but yet unexplained backward mode. (C) 2008 American Institute of Physics. [DOI: 10.1063/1.2949696]
\end{abstract}

\section{INTRODUCTION}

Space plasmas only exceptionally include a single ion species. Much more frequently, they consist of a mixture of several ion species with different charges and degrees of ionization, different masses, and concentrations, on a background of usually more mobile electrons. In the laboratory, the situation is not much different. A particularly intriguing laboratory example of a multi-ion plasma is the case of the so-called pair-ion plasma. The term "pair-ion" implies a plasma containing oppositely charged ions with nearly the same mass. In the recent past, there has been a lot of activity on the theory and the experiments dealing with such plasmas, initiated by a series of works describing the experimental production of pair ions. ${ }^{1-5}$ The ions in question are in fact, $\mathrm{C}_{60}^{ \pm}$produced in simultaneous processes of impact ionization and electron attachment, that are further collected by a magnetic filtering effect, i.e., by a diffusion in the radial direction (perpendicular to the magnetic field lines). In this way the ions are first separated from the electrons, and then collected through an annular hole (with $3 \mathrm{~cm}$ diameter), due to a subsequent downstream along the magnetic lines, in a very narrow and elongated chamber $(90 \mathrm{~cm}$ in length, and with an $8 \mathrm{~cm}$ diameter). In Refs. 2-5 several types of modes have been reported, e.g., the ion plasma wave (IPW), the ion acoustic wave (IAW), and the so-called intermediate frequency (IF) wave. Detailed measurements presented in the most recent Refs. 4 and 5, reveal that the IAW actually has two separate branches, accompanied by some additional backward propagating mode situated between them.

References 1-5 have attracted a lot of interest from researchers. This has resulted in numerous theoretical studies $^{6-16}$ dealing with various aspects of linear and nonlin- ear waves and instabilities in pair plasmas. The IF wave has been particularly in focus in a number of works, because the behavior of this mode does not fit into the standard plasma theory. Yet, the IAW also seems to be problematic because it should not appear in the perfectly antisymmetric pair-ion plasma in question here (i.e., with two ion groups with equal masses and temperatures, but opposite charges). However, specific conditions in the experiments ${ }^{1-5}$ related to the different processes (electron impact emission and attachment) involved in the production of the positive and negative ions, reveal a small disparity in the two temperatures, and this should in principle allow for an electrostatic acoustic mode to develop.

The very recent successful production of pair-hydrogen plasma $^{5}$ is even more attractive because of the small ion mass and the obvious consequences related to this. This indicates that the experimental and analytical efforts in this particular field will increase even further. Therefore, in what follows we discuss various aspects of the stability of waves in pair-ion plasmas, using some limits and parameters that are not necessarily always directed only to the presently available experimental results. This study includes various limits of the plasma density, and the presence of electrons which may naturally appear in such a plasma or can be added for the sake of scientific curiosity to check the effects introduced by their presence.

\section{MODEL}

In the experiments ${ }^{1-5}$ due to the specific filtering mentioned above, only pair ions are collected in the chamber, and it is believed that electrons are generally absent. The arguments supporting this opinion are convincing and, hence, we 
accept the mentioned filtering as a fact. Yet, electrons may be added to the pair-ion plasma in order to check the effect of their presence on the stability of the plasma. On the other hand, we note that electrons, being attached to the negative ions, still enter the chamber indirectly. Moreover, collisions enable charge exchange between the ions, formally described by a process of the type $A^{+}+B^{-} \rightarrow A+B$, and resulting in a number of neutrals in the system. The electrons can also be released from the negative ions in any of the collisions of the type $B^{-}+B \rightarrow B+B+e^{-}$, $B^{-}+A \rightarrow B+A+e^{-}$, and $A^{+}+B^{-} \rightarrow A^{+}+B+e^{-}$. As a result, a mixture of pair-ions (because in the present case $A \equiv B \equiv C_{60}$ ), electrons, and neutrals may be expected. Hence, the momentum equations for ions and electrons, that will be used below, will include the friction force between different plasma species. According to the given scenario, the presence of electrons should be related to the presence of neutrals. Yet, the neutrals are not expected to play a significant role in the system because in terms of electron collisions, roughly speaking, an ion is equivalent to $3.4 \cdot 10^{5}\left(300 / T_{e}\right)^{2}$ neutral atoms. ${ }^{17}$ So collisions between charged species are usually dominant even when the number density of neutrals $n_{n}$ by far exceeds the number density of ions. The situation is quite opposite to the presence of electrons. As a matter of fact, even a small amount of electrons can drastically change the dispersive properties of the modes in multi-ion plasmas, and indeed introduce new ones.

We note also that the spatial variation of the density in both the radial and the axial directions has been experimentally detected, ${ }^{1}$ with the radial density profile being Gaussian. In view of the very different axial and radial lengths of the chamber, the axial density inhomogeneity may be neglected without losing any essential effect. Such equilibrium density gradients are a rather common feature of various laboratory plasmas. ${ }^{18-20}$

In the experiments in Refs. $2-4$, the modes are initiated by a cylindric exciter at one end of the chamber, and they also appear to propagate almost parallel to the magnetic field vector, i.e., they may include a (small) wave number component in the perpendicular direction as well, as commented in Ref. 4.

\section{A. Unmagnetized ions}

We proceed by introducing high frequency electrostatic perturbations propagating obliquely to the equilibrium magnetic field vector $B_{0}=B_{0} \vec{e}_{z}$. In the frequency limit $\Omega_{e}>\omega \gg \Omega_{i}$, the ions are unmagnetized, the Lorentz force in the ion momentum equation can be omitted, and their perpendicular (with respect to the magnetic field vector) response to the perturbed electric field is similar to the case of an ordinary ion sound wave. In principle, the ions will remain unmagnetized even for frequencies below the ion gyrofrequency (that are in fact also experimentally measured) provided that the perpendicular wavelength is below the ion gyroradius. The momentum equation for ions of type $j$ is as follows:

$$
m_{j} n_{j} \frac{\partial \vec{v}_{j}}{\partial t}=-n_{j} q_{j} \nabla \phi-\kappa T_{j} \nabla n_{j}-m_{j} n_{j} \nu_{j} \vec{v}_{j}
$$

The electron dynamics is described by the momentum equation

$$
\begin{aligned}
m_{e} n_{e}\left[\frac{\partial \vec{v}_{e}}{\partial t}+\left(\vec{v}_{e} \cdot \nabla\right) \vec{v}_{e}\right]= & e n_{e} \nabla \phi-e n_{e} \vec{v}_{e} \times \vec{B} \\
& -\kappa T_{e} \nabla n_{e}-m_{e} n_{e} v_{e} \vec{v}_{e}
\end{aligned}
$$

and we also use the appropriate continuity equation for all species

$$
\frac{\partial n_{j}}{\partial t}+\nabla \cdot\left(n_{j} \ddot{\forall}_{j}\right)=0
$$

Here, the electron collision frequency is in principle the sum $\nu_{e}=\nu_{e i}+\nu_{e n}$.

From Eqs. (1) and (3) we get, ${ }^{21}$ for ion perturbations of the form $\sim f(x) \exp (-i \omega t+i \vec{k} \cdot \vec{r})$, where $\vec{r}$ denotes an arbitrary direction with respect to $\vec{B}_{0}$,

$$
n_{j 1}=\frac{n_{j 0} q_{j} k^{2}}{m_{j}} \frac{\phi_{1}}{\omega^{2}+i \omega \nu_{i}-k^{2} v_{T j}^{2}},
$$

where $v_{T j}^{2}=\kappa T_{j} / m_{j}$, and $\nu_{i}$ is the ion-neutral collision frequency. The electron dynamics in the perpendicular direction is described by the following recurrent formula for the velocity:

$$
\begin{aligned}
v_{e \perp}= & \frac{1}{B_{z}} \vec{e}_{z} \times \nabla_{\perp} \phi+\frac{\nu_{e}}{\Omega_{e}} \frac{\nabla_{\perp} \phi}{B_{z}}-\frac{v_{T e}^{2} \nu_{e}}{\Omega_{e}^{2}} \frac{\nabla_{\perp} n_{e}}{n_{e}} \\
& -\frac{v_{T e}^{2}}{\Omega_{e}} \vec{e}_{z} \times \frac{\nabla_{\perp} n_{e}}{n_{e}}-\frac{1}{\Omega_{e}}\left(\frac{\partial}{\partial t}+\vec{v}_{e} \cdot \nabla\right) \vec{e}_{z} \times \vec{v}_{e \perp} \\
& -\frac{\nu_{e}}{\Omega_{e}^{2}}\left(\frac{\partial}{\partial t}+\vec{v}_{e} \cdot \nabla\right) \vec{v}_{e \perp} .
\end{aligned}
$$

The parallel perturbed electron velocity is given by

$$
v_{e z 1}=-\frac{e k_{z}}{m_{e} \omega_{1}} \phi_{1}+\frac{v_{T e}^{2} k_{z}}{\omega_{1}} \frac{n_{e 1}}{n_{e 0}} .
$$

Here, $\quad \omega_{1}=\omega_{0}+i \nu_{e}, \quad \omega_{0}=\omega-k_{y} v_{e 0}, \quad$ and $\quad \vec{v}_{e 0} \approx-\left(\vec{e}_{z}\right.$ $\left.\times \nabla_{\perp} n_{e 0} / n_{e 0}\right) v_{T e}^{2} / \Omega_{e}$ is the electron diamagnetic drift. We have used $\nu_{e} \ll \Omega_{e}$ in the electron diamagnetic drift and in the last term in Eq. (5), and we work in the usual drift limit for electrons, with a small equilibrium density gradient, and in a local approximation implying $d / d x \ll k_{y}$.

\section{MODES IN COLD PLASMA}

In order to check for the most basic modes, here we omit collisions and thermal effects, and discuss first the case of a two-component electron-ion plasma. The simplified ion equation (i.e., without thermal terms ${ }^{4}$ ) is used, while the electron density perturbation becomes $n_{e 1}=\left[n_{e 0} k_{y}^{2} /\left(\Omega_{e} B_{0}\right)\right.$ $\left.-e n_{e 0} k_{z}^{2} /\left(m_{e} \omega^{2}\right)-k_{y} n_{e 0}^{\prime} /\left(\omega B_{0}\right)\right] \varphi_{1}$. From the quasineutrality condition we obtain a modified lower hybrid mode, viz., 


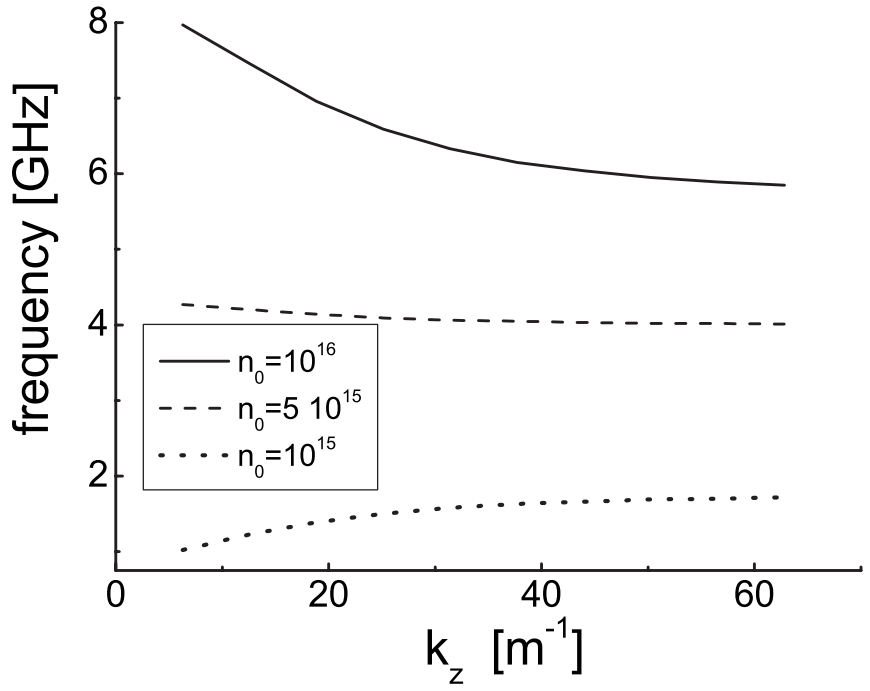

FIG. 1. The positive solution of Eq. (8) for three number densities in electron-ion plasma, showing the transition from direct to backward mode. The parameters are given in the text.

$$
\omega^{2}-\frac{\Omega_{e} n_{e 0}^{\prime}}{k_{y} n_{e 0}} \omega-\frac{k_{z}^{2} \Omega_{e}^{2}}{k_{y}^{2}}\left(1+\frac{\Omega_{i} k^{2}}{\Omega_{e} k_{z}^{2}}\right)=0 .
$$

Using the Poisson equation instead of the quasineutrality, we obtain

$$
\omega^{2}\left(1+\frac{\omega_{p p}^{2} k_{y}^{2}}{\Omega_{e}^{2} k^{2}}\right)-\frac{k_{y} \omega_{p e}^{2} n_{e 0}^{\prime}}{k^{2} \Omega_{e} n_{e 0}} \omega-\omega_{p i}^{2}-\frac{\omega_{p e}^{2} k_{z}^{2}}{k^{2}}=0 .
$$

Comparing this to Eq. (7), it is seen that the quasineutrality in the perturbed state is equivalent to assuming $\omega_{p e}^{2} k_{y}^{2} /\left(\Omega_{e}^{2} k^{2}\right) \gg 1$. For perpendicular propagation without a density gradient, Eqs. (7) and (8) yield the lower-hybrid and the ion plasma waves $\omega^{2}=\Omega_{e} \Omega_{i}$ and $\omega^{2}=\omega_{p i}^{2} /\left(1+\omega_{p e}^{2} / \Omega_{e}^{2}\right)$, respectively.

In view of the difference in mass, the last three terms in Eq. (8) cannot always be of the same order. An interesting case for which the mode behavior is determined by the density gradient term, yielding a backward solution $\partial \omega / \partial k_{z}<0$, is obtained by the following set of parameters. We take $B_{0}=0.1 \mathrm{~T}, L_{n}=n_{e 0} / n_{e 0}^{\prime}=8 \mathrm{~mm}$, and take three values for the plasma number density, omitting the index $e$ for a quasineutral equilibrium density, $n_{0}=10^{16} \mathrm{~m}^{-3}, n_{0}=5 \cdot 10^{15} \mathrm{~m}^{-3}$, and $n_{0}=10^{15} \mathrm{~m}^{-3}$. The results are presented in Fig. 1 . Here, the wave frequency has a value much above the ion gyrofrequency, but below the electron gyrofrequency, and the parallel wavelength $\lambda_{z}$ is in the range $0.1-1 \mathrm{~m}$. In the perpendicular direction we have fixed one wavelength only, i.e., $k_{y}=251 / \mathrm{m}$. The decreasing (full) line with $k_{z}$ (backward mode) represents the solution of Eq. (8) for the case of a dominant density gradient term. For a lower plasma density (dotted line) we have a direct mode $\partial \omega / \partial k_{z}>0$. In a cylindric geometry, the given solutions describe twisted waves, i.e., those traveling both along the magnetic field lines and in the $\theta$-direction. The backward mode has a behavior similar to the observed IF wave, yet in the much higher frequency domain.
The same effect, i.e., the transition from a backward to a direct mode, can be achieved and controlled by changing the perpendicular wavelength $\lambda_{y}$. This can easily be checked by setting $n_{0}=10^{16} \mathrm{~m}^{-3}$, and increasing $k_{y}$ by a factor 10 , which yields a direct mode.

It is worth mentioning that this transition from forward to backward modes by increasing the pressure, has in fact been observed experimentally in rare gases as was reported long ago in Ref. 22, but without any explanation about the source of that behavior.

We stress also that in the presence of an additional, negatively charged ion species of the same mass, the mode behavior does not change. This is because the case presented here is an interplay between the two dominant terms, i.e., the density gradient term and the last term in Eq. (8).

\section{GROWING MODES IN COLLISIONAL INHOMOGENEOUS PLASMA}

Using Eqs. (5) and (6), from the electron continuity equation we obtain

$$
\frac{n_{e 1}}{n_{e 0}}=\frac{\omega_{*}-v_{T e}^{2} k_{z}^{2}\left[1-\alpha \omega_{1} \omega_{\alpha} k_{y}^{2} /\left(k_{z}^{2} \Omega_{e}^{2}\right)\right] / \omega_{1}}{\omega-v_{T e}^{2} k_{z}^{2}\left[1-\alpha \omega_{1} \omega_{\alpha} k_{y}^{2} /\left(k_{z}^{2} \Omega_{e}^{2}\right)\right] / \omega_{1}} \frac{e T_{e}}{\kappa} .
$$

Here, $\quad \omega_{\alpha}=\omega_{0} \alpha+i \nu_{e}, \quad \alpha=1 /\left(1+\nu_{e}^{2} / \Omega_{e}^{2}\right), \quad \omega_{*}=-k_{y} \kappa T_{e} n_{e 0}^{\prime} /$ $\left(e B_{0} n_{e 0}\right)$. Note that $\omega_{0}$ appears in both $\omega_{\alpha}$ and $\omega_{1}$ from the left-hand side of the electron momentum equation, i.e., as the finite electron mass effect.

\section{A. Massless hot electrons}

In the limit $\left|\omega_{0}\right|<\nu_{e}$, from Eq. (9) we have ${ }^{23}$

$$
\frac{n_{e 1}}{n_{e 0}}=\frac{\omega_{*}+i D_{e}}{\omega+i D e} \frac{e \varphi_{1}}{\kappa T_{e}}, \quad D_{e}=\frac{k_{z}^{2} v_{T e}^{2}}{\nu_{e}}+\rho_{e}^{2} k_{y}^{2} \nu_{e}, \quad \rho_{e}=\frac{v_{T e}}{\Omega_{e}} \text {. }
$$

The second term in $D_{e}$ is small compared to the first one provided that $k_{y}^{2} \nu_{e}^{2} /\left(k_{z}^{2} \Omega_{e}^{2}\right) \ll 1$, implying a dominant effect of collisions on the parallel electron dynamics. In this case, Eq. (10) coincides with the corresponding expression from Ref. 24. The same limit holds when the electron mass corrections are retained. ${ }^{21,25}$

Using Eq. (10) instead of Eq. (9), with the quasineutrality in the equilibrium and the Poisson equation in the perturbed state, in the case of the pair-ion electron plasma with $q_{a}=-q_{b}=e$, we obtain the dispersion equation

$$
\begin{aligned}
1= & \frac{\omega_{p a}^{2}}{\omega^{2}+i \omega \nu_{a}-k^{2} v_{T a}^{2}}+\frac{\omega_{p b}^{2}}{\omega^{2}+i \omega \nu_{b}-k^{2} v_{T b}^{2}} \\
& -\frac{\omega_{p e}^{2}}{k^{2} v_{T e}^{2}} \frac{\omega_{*}+i D_{e}}{\omega+i D_{e}} .
\end{aligned}
$$

Here, $\omega_{p j}^{2}=e^{2} n_{j 0} /\left(\varepsilon_{0} m_{j}\right)$. Below we discuss several particular cases: 


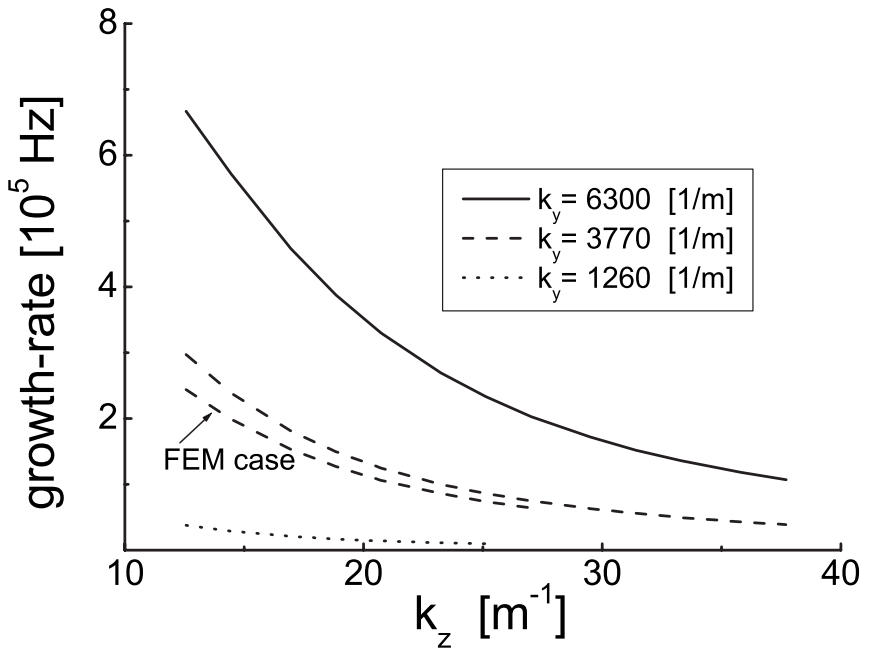

FIG. 2. The growth rate of the sound mode from Eq. (13) for three values of the perpendicular wavelengths. FEM describes the difference due to electron inertia.

(a) Without electrons and for a collisionless, pure pair-ion plasma with $m_{a}=m_{b}$, and for modes propagating along the magnetic field vector, Eq. (11) yields

$$
\omega^{2}=\omega_{p}^{2}+\frac{k_{z}^{2}\left(v_{T a}^{2}+v_{T b}^{2}\right)}{2} \pm\left[\omega_{p}^{4}+\frac{k_{z}^{4}\left(v_{T a}^{2}-v_{T b}^{2}\right)^{2}}{4}\right]^{1 / 2} .
$$

In the limit of a small $k_{z}$ (and/or a small difference between the two temperatures) this describes the ion plasma wave $\omega^{2} \simeq 2 \omega_{p}^{2}+k_{z}^{2}\left(v_{T a}^{2}+v_{T b}^{2}\right) / 2$, and the ion sound mode $\omega^{2} \simeq k_{z}^{2}\left(v_{T a}^{2}+v_{T b}^{2}\right) / 2$ when $T_{a} \neq T_{b}$. It is tempting to set $T_{a}=T_{b}$ here, however, strictly speaking, the sound mode loses its electrostatic nature in this case and it becomes an ordinary gas acoustic mode (or thermal mode) involving only the pressure perturbations.

Specific conditions in the experiments, ${ }^{1-5}$ related to different processes involved in the production of positive and negative ions (electron impact emission and attachment), reveal a small disparity in the two temperatures $T_{a} \neq T_{b}$. Hence, solving Eq. (12) with the parameters $n_{i 0}=10^{13} \mathrm{~m}^{-3}, T_{a}=0.5 \mathrm{eV}, T_{b}=0.3 \mathrm{eV}$, and for $k_{z}$ in the range $6-125 \mathrm{~m}^{-1}$, yields the ion plasma wave with frequency $\simeq 220 \mathrm{kHz}$, and the ion sound wave in the range $1.5-29 \mathrm{kHz}$. The sound mode in the experiments $^{2-5}$ appears divided into two branches. The values for the ion sound which follow from Eq. (12) are in good agreement with the lower experimental branch. However, for explaining the upper experimental branch the presence of electrons may be needed. In view of a large difference in mass, the electrons are normally described by the Boltzmann distribution. Yet, adding such a contribution of electrons only yields corrections that are of importance at spatial scales of the order of the electron Debye scale $\lambda_{\mathrm{De}}=v_{T e} / \omega_{p e}$. Therefore, extra effects are needed, like those that follow from Eq. (9), and from the last term in Eq. (11).

(b) In an electron-ion $\left(n_{b}=0\right)$ quasineutral plasma $\left(k^{2} \lambda_{\text {De }}^{2}\right.$ $\ll 1)$ with cold ions, from Eq. (11) we have

$\omega^{2}=k^{2} c_{s}^{2}\left(1+\frac{\omega-\omega_{*}}{\omega_{*}+i D_{e}}\right)$,

describing a high-frequency (as assumed $\omega>\Omega_{i}$ ) modified ion acoustic mode that may be unstable due to the density gradient and electron collisions. The numerical solution for the growth rate of the strongly growing mode (13) is presented in Fig. 2, for a relatively high (necessary to have all conditions satisfied) plasma number density $n_{e 0}=10^{19} \mathrm{~m}^{-3}$, the magnetic field $B_{0}=0.2 \mathrm{~T}$, for cold ions, and $T_{e}=5 \mathrm{eV}, L_{n}=2 \mathrm{~cm}$, and for the three values $\lambda_{y} \simeq 1,2,5 \mathrm{~mm}$. For these parameters and the given $\lambda_{y}$, we have $\omega_{*}=7.8 \cdot 10^{6}$, $4.7 \cdot 10^{6}, 1.6 \cdot 10^{6} \mathrm{~Hz}$, and the corresponding modified sound wave frequency $\omega \simeq 5 \cdot 10^{6}, 3 \cdot 10^{6}, 1 \cdot 10^{6} \mathrm{~Hz}$, respectively. The mode is growing for $\omega_{*}>\omega$. Here $m_{i}=720 m_{p}$, hence and the ions are unmagnetized. In the same time, $k^{2} \lambda_{\mathrm{De}}^{2} \ll 1$ for all three cases discussed, so that the quasineutrality is well satisfied, while $\omega_{0} / \nu_{e} \ll 1$ so that the electron inertia is negligible. The finite electron mass (FEM) line is described further in the text.

(c) In the case of pair-ion electron plasma with the Boltzmann electron response (that implies $\nu_{e} \sim \omega$ and $\left.\omega / k \ll v_{T e}\right)$, the last term in Eq. (11) is to be replaced with $\omega_{p e}^{2} /\left(k^{2} v_{T e}^{2}\right)$. Setting $T_{a}=T_{b}=T$ and $m_{a}=m_{b}=m$ yields only one pair of solutions $\omega^{2}=k^{2} c_{s}^{2}\left[T / T_{e}+\left(n_{a 0}\right.\right.$ $\left.\left.+n_{b 0}\right) / n_{e 0}\right]$ for $k^{2} \lambda_{e}^{2} \ll 1$, and $\omega^{2}=\left(\omega_{p a}^{2}+\omega_{p b}^{2}\right) k^{2} \lambda_{e}^{2} /(1$ $\left.+k^{2} \lambda_{e}^{2}\right)+k^{2} v_{T}^{2}$ for $k^{2} \lambda_{e}^{2} \geqslant 1$, where $c_{s}^{2}=\kappa T_{e} / m$, $v_{T}^{2}=\kappa T / m$.

Otherwise, from Eq. (11) for cold ions we have the third order dispersion equation

$\omega^{3}+\left(\frac{\omega_{*}}{k^{2} \lambda_{\mathrm{De}}^{2}}+i s D_{e}\right) \omega^{2}-\omega_{p a}^{2} \omega-i D_{e} \omega_{p a}^{2}=0$.

Here, $s=1+1 /\left(k^{2} \lambda_{\text {De }}^{2}\right)$. Unstable solutions may be detected by using the generalized Hurwitz method for polynomials with complex coefficients. ${ }^{26,27}$. According to this, for a polynomial of the degree $m$, of the 


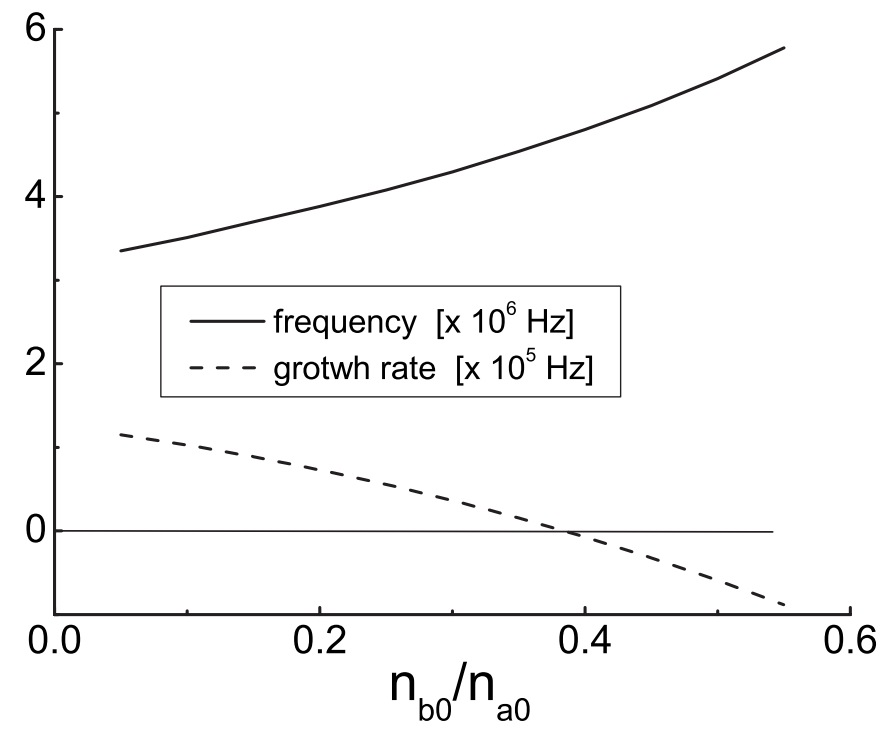

FIG. 3. The positive solution of Eq. (15) for a modified ion acoustic mode as a function of the negative-ion number density, and for equal temperatures of the two ion species.

form $\quad\left(a_{0}+i b_{0}\right) x^{m}+\left(a_{1}+i b_{1}\right) x^{m-1}+\cdots+\left(a_{m}+i b_{m}\right)=0$, one makes the sequence of $m+1$ numbers $c_{0}=a_{0}$, $c_{1}=a_{1}, \ldots, c_{r}, \ldots$, where $r$ goes to $m$, and where

$$
c_{r}=(-1)^{r(r-1) / 2}\left|\begin{array}{cccccc}
a_{1} & a_{0} & 0 & 0 & 0 & . \\
-b_{2} & -b 1 & a_{1} & a_{0} & 0 & . \\
a_{3} & a_{2} & b_{2} & b_{1} & a_{1} & . \\
-b_{4} & -b_{3} & a_{3} & a_{2} & -b_{2} & . \\
a_{5} & a_{4} & b_{4} & b_{3} & a_{3} & . \\
\cdot & \cdot & \cdot & \cdot & \cdot & . \\
\cdot & \cdot & \cdot & \cdot & \cdot & . \\
a_{2 r-1} & a_{2 r-2} & b_{2 r-2} & b_{2 r-3} & a_{2 r-3} & \cdot
\end{array}\right| .
$$

The number of roots with positive real parts equals the number of sign changes in the sequence $c_{j}$. A sufficient instability condition is that any of the $c_{r}$ has a negative sign. Applying this to Eq. (14), we find $c_{0}=1, c_{1}=\omega_{*} /\left(k^{2} \lambda_{\mathrm{De}}^{2}\right), c_{2}=-\omega_{p a}^{2} \omega_{*}^{2} /\left(k^{2} \lambda_{\mathrm{De}}^{2}\right)^{2}$, $c_{3}=-D_{e}^{2} \omega_{*}^{3} \omega_{p a}^{4} /\left(k^{6} \lambda_{\mathrm{De}}^{6}\right)$. This indicates that, for $\omega_{*}>0$, Eq. (14) has one mode propagating in the positive direction, and two solutions propagating in the opposite direction, and there exists at least one growing solution. Another possibility is $\omega_{*}<0$. According to experimental conditions $n_{e 0}^{\prime}<0$. Therefore in this case we have $k_{y}<0$, there are two sign changes in the series $c_{j}$, hence two positive solutions for $\omega$, and we still have at least one growing solution because $c_{1}, c_{2}<0$.

(d) The case (a) does not change considerably in the presence of Boltzmann electrons in the collisionless plasma and for the same parallel propagation when we have

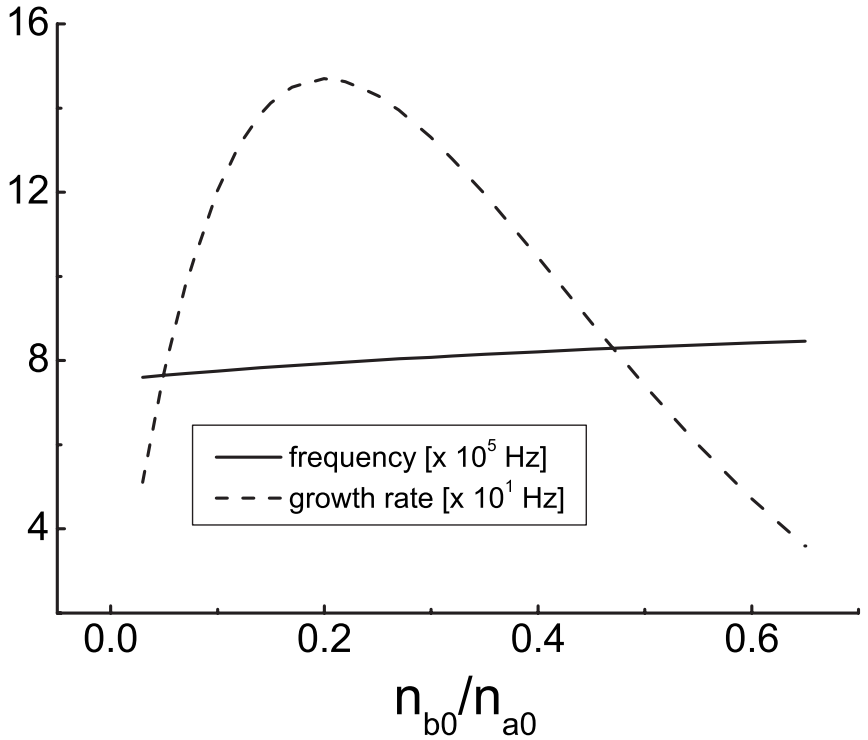

FIG. 4. Additional acoustic mode from Eq. (15) in the case of different temperatures of positive and negative ions, for $T_{a}=0.5 \mathrm{eV}$, and $T_{b}=0.3 \mathrm{eV}$.

$$
\begin{aligned}
\omega^{2}= & \frac{\omega_{p a}^{2}+\omega_{p b}^{2}}{2 s}+\frac{k_{z}^{2}}{2}\left(v_{T a}^{2}+v_{T b}^{2}\right) \pm \frac{1}{2} \\
& \times\left\{\frac{\left(\omega_{p a}^{2}+\omega_{p b}^{2}\right)^{2}}{s^{2}}+k_{z}^{2}\left(v_{T a}^{2}-v_{T b}^{2}\right)\right. \\
& \left.\times\left[\frac{2\left(\omega_{p a}^{2}-\omega_{p b}^{2}\right)}{s}+k_{z}^{2}\left(v_{T a}^{2}-v_{T b}^{2}\right)\right]\right\}^{1 / 2} .
\end{aligned}
$$

This is just a modified form of Eq. (12).

\section{B. Full dispersion equation}

Keeping the full form of Eq. (9), with the help of the Poisson equation, the dispersion equation becomes

$$
\begin{aligned}
1= & \frac{\omega_{p a}^{2}}{\omega^{2}+i \omega \nu_{a}-k^{2} v_{T a}^{2}}+\frac{\omega_{p b}^{2}}{\omega^{2}+i \omega \nu_{b}-k^{2} v_{T b}^{2}} \\
& -\frac{1}{k^{2} \lambda_{e}^{2}} \frac{\omega_{*}-v_{T e}^{2} k_{z}^{2}\left[1-\alpha \omega_{1} \omega_{\alpha} k_{y}^{2} /\left(k_{z}^{2} \Omega_{e}^{2}\right)\right] / \omega_{1}}{\omega-v_{T e}^{2} k_{z}^{2}\left[1-\alpha \omega_{1} \omega_{\alpha} k_{y}^{2} /\left(k_{z}^{2} \Omega_{e}^{2}\right)\right] / \omega_{1}} .
\end{aligned}
$$

Equation (15) will be solved numerically for some sets of parameter values.

We first check the effects of the finite electron mass (FEM), and solve Eq. (15) for the electron-ion case $\left(n_{b 0}=0\right)$ with the same parameters as in Fig. 2, for cold ions, and for the case $k_{y}=3770 \mathrm{~m}^{-1}$ (dashed line). The sound wave frequency appears to be slightly larger, i.e., in the range 3.1-3.2 MHz, while the growth rate is reduced, given by the lower dashed line in Fig. 2.

For the pair-ion electron plasma, Eq. (15) is solved for collisionless ions in terms of $n_{b 0} / n_{a 0}$, and the result for the acoustic mode is presented in Fig. 3 for the same parameters as in Fig. 2 and for fixed values of $k_{z}=18.8 \mathrm{~m}^{-1}$ and $k_{y}=3770 \mathrm{~m}^{-1}$. The driving source for the instability is the density gradient in combination with electron collisions. Hence, for a reduced electron number density the instability 
vanishes; in the present case this happens at $n_{b 0} / n_{a 0} \simeq 0.4$. The other pair of solutions, that is in the range of the ion plasma frequency and the lower hybrid frequency, appears strongly damped, with $\omega_{r}$ being of the same order as $\left|\omega_{i}\right|$ and it is consequently of no interest.

For much lower density, e.g., setting $n_{a 0}=10^{14} \mathrm{~m}^{-3}$ and varying $n_{b 0} / n_{a 0}$, we obtain two direct modes with frequencies close to $10^{6} \mathrm{~Hz}$ and $2 \cdot 10^{7} \mathrm{~Hz}$, propagating almost without change, the former very weakly growing and the latter being almost undamped.

In the case of different temperatures of two pair-ion species (like in the reported cases in Refs. 2-5), there appears an additional acoustic mode. This is checked by setting $T_{a}=0.5 \mathrm{eV}, T_{b}=0.3 \mathrm{eV}$ for $n_{a 0}=10^{19} \mathrm{~m}^{-3}$. The other parameters are the same as in Fig. 3. In total, there are three pairs of solutions: one high frequency $\sim 10^{7} \mathrm{~Hz}$ but highly damped mode mentioned above, the slightly modified acoustic mode from Fig. 3, and the third, weakly growing acoustic mode presented in Fig. 4.

We stress that the negative solutions (that are not presented in the figures above) are in fact not counterpart solutions in the strict sense because of the asymmetry caused by the density gradient.

\section{WEAKLY MAGNETIZED IONS}

In the experiments, ${ }^{2-5}$ the ions in the equilibrium are magnetized. However, for perturbations with frequencies above the ion gyrofrequency, the ions are unmagnetized, yet, for not so high frequency oscillations they can feel the effects of the magnetic field. Hence, in Eq. (1) we add the Lorentz force. The equilibrium diamagnetic drift velocity for the two ion species is given by $\vec{v}_{j 0}=\left(\vec{e}_{z} \times \nabla n_{j 0} / n_{j 0}\right) \kappa T_{j} /\left(q_{j} B_{0}\right)$. For the given experimental parameters $\left(T_{a}=0.5 \mathrm{eV}, T_{b}=0.3 \mathrm{eV}\right)$ and assuming $L_{n}$ of the order of $1 \mathrm{~cm}$, the two velocities are of the order of $10^{2} \mathrm{~m} / \mathrm{s}$. For perpendicular wavelengths of the order of $1 \mathrm{~cm}$, the Doppler shift in the frequency, $k_{y} v_{j 0}$, becomes of the order $10^{5} \mathrm{~Hz}$, and can therefore introduce visible effects in the mode behavior. Assuming a negative density gradient, we have a negative $v_{a 0} \vec{e}_{x}$ and $\vec{v}_{b 0}=-\vec{v}_{a 0} T_{b} / T_{a}$. The velocity components for the ion species $a$ become

$$
\begin{aligned}
& v_{a x 1}=\frac{i}{\omega_{a 0}^{2}-\Omega^{2}}\left[k_{y} \Omega^{2} \frac{\phi_{1}}{B_{0}}+v_{T a}^{2}\left(k_{y} \Omega-\omega_{a 0}\left|\frac{n_{a 0}^{\prime}}{n_{a 0}}\right|\right) \frac{n_{a 1}}{n_{a 0}}\right], \\
& v_{a y 1}=\frac{1}{\omega_{a 0}^{2}-\Omega^{2}}\left[k_{y} \omega_{a 0} \Omega \frac{\phi_{1}}{B_{0}}+v_{T a}^{2}\left(k_{y} \Omega-\omega_{a 0}\left|\frac{n_{a 0}^{\prime}}{n_{a 0}}\right|\right) \frac{n_{a 1}}{n_{a 0}}\right], \\
& v_{a z 1}=\frac{k_{z} \Omega}{\omega_{a 0}} \frac{\phi_{1}}{B_{0}}+\frac{k_{z} v_{T a}^{2}}{\omega_{a 0}} \frac{n_{a 1}}{n_{a 0}} .
\end{aligned}
$$

Here, $\omega_{a 0}=\omega-k_{y} v_{a 0}$. In the corresponding equations for the ion species $b$, the term $\Omega$ is to be taken with the minus sign.

The continuity equation $n_{j 1} / n_{j 0}=\left(k_{y} v_{j y 1}+k_{z} v_{j z 1}\right.$ $\left.+i v_{j x 1}\left|n_{0}^{\prime} / n_{0}\right|\right) / \omega_{j 0}$ for the two species, and the Poisson equation, yield the dispersion equation for the modes in an inhomogeneous pair-ion plasma,

$$
\begin{aligned}
\frac{k^{2}}{\omega_{p}^{2}}=\frac{\frac{k_{y}^{2}}{\omega_{a 0}^{2}-\Omega^{2}}+\frac{k_{z}^{2}}{\omega_{a 0}^{2}}-\left|\frac{n_{a 0}^{\prime}}{n_{a 0}}\right| \frac{k_{y} \Omega}{\omega_{a 0}\left(\omega_{a 0}^{2}-\Omega^{2}\right)}}{1-\frac{k_{z}^{2} v_{T a}^{2}}{\omega_{a 0}^{2}}-\frac{k_{y}^{2} v_{T a}^{2}}{\omega_{a 0}^{2}-\Omega^{2}}\left(1-\frac{1}{\omega_{a 0}} \frac{k_{y}}{k_{a 0}^{\prime}} \mid\right)+\left|\frac{n_{a 0}^{\prime}}{n_{a 0}}\right| \frac{v_{T a}^{2}}{\omega_{a 0}^{2}-\Omega^{2}}\left(\frac{k_{y} \Omega}{\omega_{a 0}}-\left|\frac{n_{a 0}^{\prime}}{n_{a 0}}\right|\right)} \\
+\frac{\frac{k_{y}^{2}}{\omega_{b 0}^{2}-\Omega^{2}}+\frac{k_{z}^{2}}{\omega_{b 0}^{2}}+\left|\frac{n_{a 0}^{\prime}}{n_{a 0}}\right| \frac{k_{y} \Omega}{\omega_{b 0}\left(\omega_{b 0}^{2}-\Omega^{2}\right)}}{1-\frac{k_{z}^{2} v_{T b}^{2}}{\omega_{b 0}^{2}}-\frac{k_{y}^{2} v_{T b}^{2}}{\omega_{b 0}^{2}-\Omega^{2}}\left(1+\frac{\Omega}{\omega_{b 0}} \frac{1}{k_{y}}\left|\frac{n_{a 0}^{\prime}}{n_{a 0}}\right|\right)-\left|\frac{n_{a 0}^{\prime}}{n_{a 0}}\right| \frac{v_{T b}^{2}}{\omega_{b 0}^{2}-\Omega^{2}}\left(\frac{k_{y} \Omega}{\omega_{b 0}}+\left|\frac{n_{a 0}^{\prime}}{n_{a 0}}\right|\right)} .
\end{aligned}
$$

In the absence of electrons, the density gradients and number densities are the same, and $\omega_{p}^{2}=e^{2} n_{0} /\left(\varepsilon_{0} m\right)$. Various limits of Eq. (16) can easily be discussed, yielding the previously discussed modes. For example, in the absence of a density gradient and for strictly perpendicularly propagating oscillations in a quasineutral plasma, from Eq. (16) we have just the ion cyclotron mode $\omega^{2}=\Omega^{2}+k^{2}\left(v_{T a}^{2}+v_{T b}^{2}\right) / 2$. For strictly parallel propagation in a quasineutral plasma we have the acoustic mode $\omega^{2}=k^{2}\left(v_{T a}^{2}+v_{T b}^{2}\right)$, otherwise it yields Eq. (12).

A particularly interesting limit is seen also in the case of a cold plasma and for long wavelengths, when the remaining density gradient terms in the numerators in Eq. (16) exactly cancel each other, and it yields electrostatic convective cells $\omega^{2}=\Omega^{2} k_{z}^{2} /\left(k_{y}^{2}+k_{z}^{2}\right)$. This result is completely different compared to the standard electron-ion plasma which in such a geometry has the drift wave driven by the density gradient.

Equation (16) is solved numerically for parameters close to the experimental values. We take $B_{0}=0.2 \mathrm{~T}, T_{a}=0.5 \mathrm{eV}$, $T_{b}=0.3 \mathrm{eV}$, but with a lower number density $n_{a 0}=n_{b 0}$ $=10^{12} \mathrm{~m}^{-3}$, and we take $L_{n}=0.8 \mathrm{~cm}$. The result for modes with positive frequency is presented in Fig. 5. For values of $k_{z}$ in the range $0.01-1.15$ (in the given units), the ion plasma mode is in fact a backward one (frequency decreases from 14.05 at $k_{z}=0.01$ to 12.7 at $\left.k_{z}=0.15\right)$, and these values are 


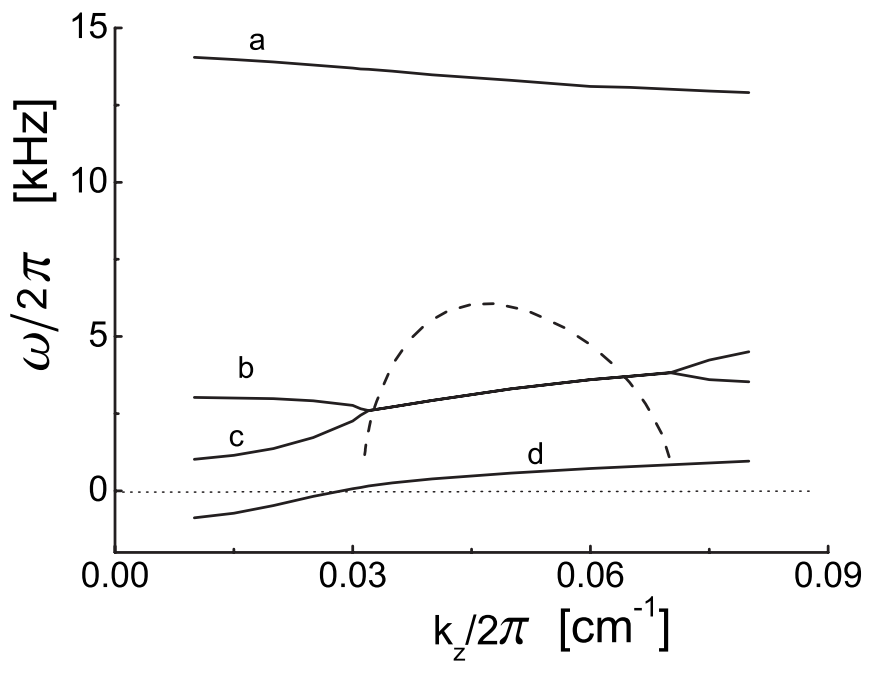

FIG. 5. The positive numerical solutions of Eq. (16) for a pure pair- ion plasma. The growth rate of the coupled modes (multiplied by 10) is given by the dashed line.

only slightly below the experimental values that correspond to the IF mode. However, we can obtain exactly the same frequency as for the observed IF mode by simply slightly increasing the density to the value $n_{a 0}=n_{b 0}=3 \cdot 10^{12} \mathrm{~m}^{-3}$, but then the decrease with $k_{z}$ in this case becomes less pronounced, though it still remains there. We suspect that the unexplained experimental IF mode may in fact be the ion plasma mode presented here, while the experimental upper mode (which in Refs. 2-5 has been interpreted as the ion plasma mode) may be an eventual contribution of electrons, described by our Eqs. (9) and (10). However, these statements are speculative as we have not been able to obtain an additional electron mode in the given experimental range, i.e., at frequencies about 30 and higher (in the units given in Fig. 5).

Due to the density gradient there is a coupling between modes $b$ and $c$, in the range $k_{z} \approx 0.032-0.07$, with the maximum growth rate $\omega_{i}$ of the order $\omega_{i} / \omega_{r} \simeq 0.2$. The corresponding growth rate, multiplied by 10 , is presented by dashed line in Fig. 5. The density gradient modifies also the acoustic mode $d$ so that for small values $k_{z}$ its frequency becomes negative. This all can easily be demonstrated by switching of the density gradient terms.

We note that the line $b$ has a range of $k_{z}$ for which it is backward $\partial \omega / \partial k_{z}<0$, and the same holds for the line $c$. The frequency of both modes, in the range where they have backward properties, is in the domain of the low frequency backward mode observed in the experiment (around 4 in the given units). Though, the agreement with experimental solutions is far from perfect, and the splitting of the lines $b$ and $c$ we obtain for $k_{z}>0.07$ has indeed not been observed.

\section{CONCLUSIONS}

The pair-ion plasma containing heavy fullerene ions (with equal positive and negative charge), that has been created in the laboratory, has attracted a lot of interest in the recent past. The present study deals with some additional properties of such plasmas, e.g., those that result from the eventual presence of electrons, from plasma inhomogeneity combined with electron collisions, and from much higher densities.

The arguments given in Sec. II show that the presence of electrons cannot be excluded, or that they can be added to the system in order to check the consequences of their presence. In a recent study ${ }^{9}$ some properties of waves in pair ion plasma have been discussed, showing that in fact they can be used as an indicator of the presence of electrons. Some indications in this direction can also be found in the most recent experimental results, ${ }^{4,5}$ where the measured sound branch in fact splits into two sub-branches. In the present work, the effects of the density gradient and electron collisions in a relatively high density pair-ion plasma have been explored showing the presence of a backward-propagating highfrequency mode with features similar to the observed IF mode that remained unexplained so far. However, our first backward mode, determined by the density gradient (see Fig. 1), appears in a much higher frequency range and it is therefore unable to explain the observed IF mode. The case of a pure pair-ion plasma, discussed in Sec. V, reveals that the ion plasma wave behaves as a backward mode in a large range of the parallel wave number, and perhaps may be interpreted as the IF mode observed in the experiment. Yet, the agreement with experimental modes is not good enough to draw a definite conclusion. Nevertheless, in our view, the plasma features discussed here represent a step forward in the theory of such a newly created plasma. At the same time we believe that the presented results should make a good basis for future investigations directed towards the exact explanation of the IF mode.

\section{ACKNOWLEDGMENTS}

The results presented here are obtained in the framework of Project Nos. G.0304.07 (FWO-Vlaanderen), C 90205 (Prodex), GOA/2004/01 (K.U.Leuven), and the Interuniversity Attraction Poles Programme-Belgian State-Belgian Science Policy.

${ }^{1}$ W. Oohara and R. Hatakeyama, Phys. Rev. Lett. 91, 205005 (2003).

${ }^{2}$ W. Oohara and R. Hatakeyama, Phys. Rev. Lett. 95, 175003 (2005).

${ }^{3}$ R. Hatakeyama and W. Oohara, Phys. Scr. 116, 101 (2005).

${ }^{4}$ W. Oohara, Y. Kuwabara, and R. Hatakeyama, Phys. Rev. E 75, 056403 (2007).

${ }^{5}$ W. Oohara and R. Hatakeyama, Phys. Plasmas 14, 055704 (2007).

${ }^{6}$ J. Vranjes and S. Poedts, Plasma Sources Sci. Technol. 14, 485 (2005).

${ }^{7}$ H. Schamel and A. Luque, New J. Phys. 7, 69 (2005).

${ }^{8}$ A. Luque, H. Schamel, B. Eliasson, and P. K. Shukla, Phys. Plasmas 12, 122307 (2005).

${ }^{9}$ H. Saleem, J. Vranjes and S. Poedts, Phys. Lett. A 350, 375 (2006).

${ }^{10}$ I. Kourakis, A. Esfandyari-Kalejahi, M. Medhipoor, and P. K. Shukla, Phys. Plasmas 13, 052117 (2006).

${ }^{11}$ A. Esfandyari-Kalejahi, I. Kourakis, and P. K. Shukla, Phys. Plasmas 13, 122310 (2006).

${ }^{12}$ A. Luque, H. Schamel, B. Elisasson, and P. K. Shukla, Plasma Phys. Controlled Fusion 48, L57 (2006).

${ }^{13}$ F. Verheest, Phys. Plasmas 13, 082301 (2006).

${ }^{14}$ H. Saleem, Phys. Plasmas 13, 044502 (2006); 14, 014505 (2007).

${ }^{15}$ B. Zhao and J. Zheng, Phys. Plasmas 14, 062106 (2007).

${ }^{16}$ I. Kourakis, F. Verheest, and N. F. Cramer, Phys. Plasmas 14, 022306 (2007).

${ }^{17}$ J. A. Ratcliffe, The Magneto-Ionic Theory and its Applications to the Ionosphere (Cambridge University Press, Cambridge, 1959), p. 33. 
${ }^{18}$ J. Vranjes, A. Okamoto, S. Yoshimura, S. Poedts, M. Kono, and M. Y. Tanaka, Phys. Rev. Lett. 89, 265002 (2002).

${ }^{19}$ A. Okamoto, K. Hara, K. Nagaoka, S. Yoshimura, J. Vranjes, M. Kono, and M. Y. Tanaka, Phys. Plasmas 10, 2211 (2003).

${ }^{20}$ O. Grulke, F. Greiner, T. Klinger, and A. Piel, Plasma Phys. Controlled Fusion 43, 525 (2001).

${ }^{21}$ J. Vranjes, M. Y. Tanaka, and S. Poedts, Phys. Plasmas 13, 122103 (2006).
${ }^{22}$ P. J. Barett and P. F. Little, Phys. Rev. Lett. 14, 356 (1965).

${ }^{23}$ A. B. Mikhailovskii, Theory of Plasma Instabilities (Consultants Bureau, New York, 1974), Vol. 2, p. 192.

${ }^{24} \mathrm{~J}$. Weiland, Collective Modes in Inhomogeneous Plasmas (Institute of Physics, Bristol, 2000), p. 32.

${ }^{25}$ J. Vranjes, B. P. Pandey, and S. Poedts, Phys. Plasmas 14, 032106 (2007).

${ }^{26}$ D. L. Giaretta, Astron. Astrophys. 75, 237 (1979).

${ }^{27}$ J. Vranjes and S. Poedts, Astron. Astrophys. 458, 635 (2006). 